\section{Durable, colour-stable hybrid ceramics}

Whether aesthetic anterior restorations or inlays, onlays and posterior crowns with high colour and surface stability: Shofu Block $\mathrm{HC}$ can be used for almost all indications for modern CAD/CAM technology and machined wet or dry in all standard milling units. Shofu has added two-layer blanks to its line of hybrid ceramics and fitted blocks with universal holding pins. So Shofu Block HC, a highperformance $\mathrm{CAD} / \mathrm{CAM}$ material, is now available in three types (Universal, Cerec and Ceramill) and as one and two-layer blocks. Users may choose from various high and low-translucency shades and two enamel shades, designed to reliably meet every need in the field of all-ceramic restorations.

The excellent physical properties of Shofu Block HC enable the creation of crowns and implant-supported restorations characterised by great durability and absorption of occlusal forces. Moreover, highly aesthetic restorations can be created thanks to enamel-like light transmission and the addition of two-layer blocks featuring a smooth, natural shade transition from dentine to enamel.

In combination with the HC Primer, which ensures very high bond strengths thanks to its unique infiltration effect, and SHOFU's gentle polishing and luting systems, a CAD/CAM restoration system with perfectly matched components is provided - for all modern milling units, aesthetic requirements and clinical indications.

For further information, contact the Shofu office 01732783580 or sales@shofu.co.uk.

\section{An infection control show-stopper}

CleanCert is delighted to be exhibiting at this year's Dentistry Show on stand H100.

CleanCert's unique, innovative dental infection control and water purification products will be available to view throughout the event, including:

- CleanCert+ biofilm disinfectant- a revolutionary one-stage waterline disinfectant that is independently proven to be efficacious, as well as safe and simple to use

- Reverse osmosis: a fast, easy and cost-effective system for providing as much purified water as the dental practice needs, on tap

- LabCert: a highly effective and fast-acting disinfectant, which can be employed on all materials and appliances that are transferred between dental practices and laboratories, ensuring the wellbeing of staff and patients alike

- Performance-monitoring dipslides and software apps.

To learn more about these exciting advances in infection control, visit stand $\mathrm{H} 100$, where you can be sure of a warm welcome. Plus free samples to take away.

Alternatively, visit cleancert.co.uk, email sales@cleancert.co.uk or call 08443511115.
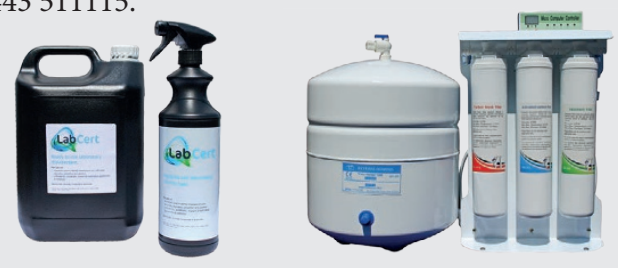

\section{Preparing for enhanced data protection}

\section{Simplyhealth Professionals has} produced a range of templates and draft policies to support its practices in preparation for meeting the enhanced data protection require-

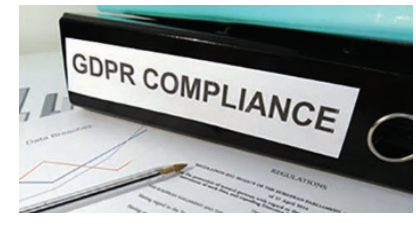
ments coming into force on 25 May 2018. It has also been providing detailed information and guidance on the implication for practices of the new data regulations with a three part blog written by Roger Matthews, Honorary Life President and former Chief Dental Officer (https://www.denplan.co.uk/dentists/blog).

Between now and 25 May, the recommendations are that practices will need to:

- Complete their data audit (as recommended by The Information Commissioner's Office (www.ico.org.uk/gdpr)

- Check where back-ups are stored (ask your software provider/s)

- Consider how to present Privacy Notices to patients

- Consider revising their Data Protection and Information Security policies

- Update their Cookie policy if they have a website

- Carry out and document a Legitimate Interest Assessment (in simple terms how you lawfully process personal data)

- Draw up a Data Breach policy and procedure (if not already done)

- Appoint a Data Protection Officer.

To help with preparation, Simplyhealth has published several templates for members on their web portal in a GDPR toolkit. There are templates available for a Legitimate Interest Assessment, a Privacy Notice and a Data Breach. In each case it will be necessary for practices to consider how these templates should be adapted for their own particular circumstances and practice. Further resources will be published on the portal in the coming weeks in the form of a Cookie policy, a Data Retention policy, a Data Protection policy and an Information Security policy.

As the new law is still a Parliamentary 'work in progress' and subject to some further amendments, Simplyhealth Professionals intends to keep members fully updated on any further developments.

\section{Webinar highlights the impact of modern diets and lifestyle on dentition}

GSK are inviting dental professionals to view a recording of their recent panel webinar on the topic of enamel wear with a particular focus on younger people and practical advice for this category. The panel was chaired by Dr Rupert Austin, specialist in prosthodontics, and he was joined by Dr Manrina Rhoda (cosmetic dental surgeon), Ali Lowe (dental hygienist) and Amit Rai (GDP).

To access the full webinar footage and gain one hour of verifiable CPD visit https://digital.vevent.com/rt/ gskpronamel $\sim$ webinar and register to view.

A desire for healthy lifestyles was identified as a potential reason for the increase in levels of tooth wear amongst younger patients. Habits including drinking hot water with lemon or snacking on fruit, vegetables and juices can all play a part. It was also highlighted that the stresses of modern life have led to increased signs of bruxism and tooth grinding in practice. 